\title{
Response to commentary by Grifell and Hart, commentary on: The problem of mephedrone in Europe: Causes and suggested solutions
}

We would like to thank Grifell and Hart for commenting on our latest article.

Firstly, in our article we write about the increase in the frequency of admissions of patients hospitalized due to taking mephedrone on a regular basis. The evidence for this can be found in our article published in The Lancet Psychiatry. Between 2010 and 2018,601 patients on a mephedrone binge were hospitalized in the Nowowiejski Hospital in Warsaw. In each successive year, an increasing number of patients were hospitalized for this reason [1].

Secondly, regarding the increase in mortality associated with mephedrone intake, this problem has been signaled using the example of London in an article published in The Lancet. The authors indicate that a larger number of patients died due to mephedrone intake in London in 2015 compared to 2014 [2]. This may be due to the fact that almost all people who take mephedrone combine it with other psychoactive substances. In our study, conducted in the Nowowiejski Hospital in Warsaw, 99.5\% of people on a mephedrone binge combined mephedrone with other psychoactive substances, primarily alcohol, heroin and benzodiazepines. In general, in recent months in Poland there have been numerous cases of death caused by the consumption of legal highs. These people usually took many psychoactive substances at the same time [1].

Thirdly, almost $20 \%$ of the 601 people on a mephedrone binge examined by us in the Nowowiejski Hospital in Warsaw were again sent to a psychiatric hospital for the same reason, in some cases there were even seven consecutive hospitalizations. This indicates the strong addictive potential of mephedrone. This is also confirmed by entries on special internet forums, such as hyperreal.info, where people taking mephedrone indicate its strong addictive potential [1,3].

Fourthly, we agree with the authors of the commentary on our article that limiting the availability of one compound may lead to the appearance of new psychoactive substances. However, this view is quite controversial given the fact that new substances are constantly appearing, regardless of whether action is taken to reduce the availability of existing substances. It is also true that mephedrone is often advertised and sold as a chemical reagent, bath salt, plant fertilizer and fragrance freshener for vacuum cleaners but it is, in fact, a different substance. This contributes to an increase in the hospitalization of patients taking substances advertised as mephedrone and increases the risk of death (this situation does not seem to have occurred in the cases of people we examined in whom mephedrone was found with laboratory tests). We have, therefore, highlighted in our article, based on the example of mephedrone, what solutions can be proposed. New psychoactive substances can be synthetized if the sale of the existing one is legally banned, yet this can be affected by poor education on the subject. We should even more strongly highlight the addictive potential of highs (including mephedrone), the longterm effects of their intake, as well as the consequences of combining them with other psychoactive substances. The recently deceased Polish neuroscientist Professor Jerzy Vetulani wrote: 'The trouble is that addictive substances are still a bit taboo. The attitude of at least part of society is extremely hostile. How are teenagers educated about drugs? They are usually educated by being scared. These bugbears are supposed to distract them from drugs. However, this will only deter those who would not be interested in them anyway...' [4]. For this reason, it seems necessary to add many important elements to education including the issue of the simultaneous use of many psychoactive substances. Perhaps this would reduce the likelihood of creating and adopting newer and more dangerous psychoactive substances. It is also necessary to run educational campaigns, including by the scientific community, and to block websites promoting mephedrone.

In conclusion, we thank the authors very much for sending us their valuable commentary. We are attaching our article that shows the problem of the increase in the frequency of admissions of patients on a mephedrone binge to the psychiatric hospital in Warsaw between 2010 and 2018. We agree that it is necessary to implement the same rigorous evidence-based system throughout Europe, otherwise the problem will become even more serious.

\section{References}

[1] Ordak M, Nasierowski T, Muszynska E. The growing problem of mephedrone use in Warsaw, Poland, 2010-18. Lancet Psychiatry 2018;5(10):787. 
[2] Hockenhull J, Murphy KG, Paterson S. Mephedrone use is increasing in London. Lancet 2016;387:1719-20.

[3] Ordak M, Nasierowski T, Bujalska-Zadrozny M. The problem of mephedrone in Europe: causes and suggested solutions. Eur Psychiatry 2019;55:43-4.

[4] Vetulani J. Beauty of neurobiology. Homini 2014;79-86.

M. Ordak* Department of Pharmacodynamics, Centre for Preclinical Research and Technology (CePT), Medical University of Warsaw, St. Banacha

1B, 02-097 Warsaw, Poland
T. Nasierowski Department of Psychiatry, Medical University of Warsaw, Poland

* Corresponding author. E-mail address: mordak@wum.edu.pl (M. Ordak).

Received 15 November 2018

Available online 28 November 2018 